\title{
The Relationship between Dividend Payout and Price-to-Earnings
}

\author{
CNV Krishnan \\ Case Western Reserve University \\ Yifei Chen \\ Case Western Reserve University
}

Using a large database of S\&P 1500 index firms spanning an 88-quarter period from 1995 through 2016 , we document that market cap (firm size), book-to-market ratio (a proxy for market perception of inverse of growth potential) and industry matter for determining Price-to-Earnings (PE) levels as a function of payout levels: current-period dividend payout is significantly and positively correlated with next period $P E$ ratio for high market cap firms, and significantly and negatively correlated for high book-to-market firms. However, once the PE levels are determined, current period dividend payout change is significantly and negatively associated with next period PE change. We show that an increase in current period payout signals reduced investment opportunities and increased risk, which reduce PE ratios.

Keywords: Price-to-Earnings ratio, PE ratio, dividend payout ratio, industry, market cap, firm size, bookto-market ratio, growth opportunities, firm risk.

\section{INTRODUCTION}

Investors focus on Price-to-Earnings (PE) ratios, often classifying firms into high and low PE stocks. Hough (2011), for example, argues that low PE stocks outperformed high PE stocks in the 2000's. Standard text book models (See, for example, Professor Damodaran's online teaching notes at http://pages.stern.nyu.edu/ adamodar) show that the PE ratio can be stated in terms of expected earnings in the next time period:

$$
\frac{\mathrm{P}_{0}}{\mathrm{EPS}_{1}}=\text { Forward } \mathrm{PE}=\frac{\text { Payout Ratio }}{k_{e}-g_{k}}
$$

The PE ratio is, then, an increasing function of the payout ratio and the growth rate, and a decreasing function of firm risk. We empirically examine whether such a standard model holds in the cross-section of firms. In particular, we empirically analyze the determinants of both the level of, and changes to the PE ratio.

Extant literature has studied both PE ratios and dividend payouts extensively. Risk could matter: Henne, Ostrowski and Reichling (2009) argue that stock performance generally improves with increasing dividend yield, but this result is based on risk reduction rather than higher return, in the German market. Ferson (2008) argues that an argument that a shock to expected return on equity changes asset value may 
overstate the effect to the extent that a shock that changes the required return also changes the expected future cash flows. Growth could matter: Ang and Zhang (2011) find that growth opportunities account for approximately $95 \%$ of the variation in PE ratios, and $80 \%$ of the level of PE ratios. Riahi-Belkaoui and Picur (2001) allege that firms with high investment opportunities are "PE valued". Profitability could matter: Benartzi, Michaely, and Thaler (1997) and Grullon, Michaely, and Swaminathan (2002) find no evidence that dividend changes predict abnormal increases in earnings. Penman (1996) fails to find that the current return on equity is a good indicator of PE ratio. Our goal in this paper is to use a large panel data of firm-quarters, across different industries, market cap, and growth, to analyze the determinants of $\mathrm{PE}$ ratio levels and changes, in particular, as functions of dividend payout levels and changes.

We start with examining the determinants of PE levels, and find, in univariate tests, that industry matters. High-tech and healthcare firms have higher PE ratios, on average, and manufacturing firms the lowest, broadly reflecting future growth opportunities. Market capitalization (which is a proxy for firm size) and book-to-market ratio (which is a proxy for (the lack of) growth options) matter. The higher the market capitalization, the higher the PE, showing the market prices in market dominance, and the lower the book-to-market ratio the higher the PE, reflecting future growth opportunities.

In multivariate regressions, after controlling for industry and time fixed effects, we find that current period dividend payout is significantly and positively related to next period PE. Results are significant when we use current earnings or trailing earnings. When we run regressions on groups of firms divided by industries, market cap, book-to-market ratio and recession and non-recession years, we find that results are more complicated. For Consumer and High Tech industries, the correlation between current period payout and next period PE tends to be significantly negative, while it is significantly positive for Manufacturing and other industries. In general, we find a positive relationship between current period payout and future PE for high market cap firms. So, a simple dividend discount model is perhaps too simple to use across all industry segments, market capitalization, book-to-market ratios, and other measures of firm risk.

When we examine changes, the change in PE ratio is significantly and negatively associated with prior changes in payout. This negative relationship not only appears overall, but also in almost every industry and type of companies: all of the significant associations between current period change in payout and future change in PE ratio are negative. We examine the impact of payout changes on risk, and find that there is a significant and positive relationship. Thus, given PE levels, an increase in current period payout signals reduced growth opportunities and increased risk, and hence results in a decrease in next period PE.

\section{DATA AND DESCRIPTIVE STATISTICS}

We use all S\&P 1500 firms' (including S\&P 500, S\&P Mid Cap 400 and S\&P Small Cap 600) data from the Compustat Quarterly from 1995 through 2016, entailing 88 quarters and 132,000 observations in all.

We define book equity (BE) as total shareholders' equity plus deferred taxes and investment tax credit (item TXDITCQ) minus the book value of preferred stock (item PSTKQ). We prefer the shareholders' equity numbers (SEQQ). In case this data are not available, we calculate shareholders' equity as sum of common and preferred equity (items CEQQ and PSTKQ). If neither of the two are available, we define shareholders' equity as the differences of total assets and total liabilities (items ATQ and LTQ). Trailing earnings is the average net income of the past four quarters. We use 5 industry groups defined by Ken French, which are Consumer, Manufacturing, High Technology, Healthcare and Other (see http://mba.tuck.dartmouth.edu/pages/faculty/ken.french/data_library.html).

We also segregate years by recession and non-recession years. The definition of recession years is taken from the Business Cycle Dating Committee of the National Bureau of Economic Research (NBER) based on the behavior of various indicators of economic activity. The list of variables used is shown in Appendix. 


\section{UNIVARIATE RESULTS}

Figure 1 and Figure 2, respectively, show the time series plots of quarterly average dividend payout and quarterly average PE overall, as well as by industries, by market capitalization and by book-to-market categories, where the recession quarters 2001 Q2 - 2001 Q4 and 2008 Q1 - 2009 Q2, as determined by NBER, are shaded. Panel A of Figure 1 shows that dividend payout ratio tends to spike up during recession periods. Panel B shows that manufacturing firm payouts tend to be higher, Panel C shows the higher the market cap the greater the dividend payout, while the final panel D shows that the higher the book to market ratio, the higher the payout. This is confirmed by the pairwise differences in Table 1 . Manufacturing firms have significantly higher payout than other firms. Healthcare and high-tech firms have the lowest payouts on average, perhaps reflecting their future growth potential. The middle panel shows the higher the market capitalization the higher the payout reflecting firm maturity, while the last panel shows that the higher the book-to-market ratio the higher the payout reflecting lack of growth potential. So, in summary, manufacturing firms payout more, larger firms payout more, and firms with lower future growth potential payout more, on average.

Panel A of Figure 2 shows that PE ratios, on average, tend to be lower during recessions because of price depressions. There is no clear pattern for PE ratios emerging when we look by industries, although healthcare stocks in recent years tend to have higher PE ratios. However, Panel C shows that low market cap firms (small firms) generally tend to have lower PE ratios, while Panel D shows that the higher the book to market ratio, the lower the PE ratio, reflecting the fact that the lower the future growth prospects the lower the PE ratio. This is corroborated by the pairwise differences among the groups of firms by industries, market capitalization and book to market in Table 2. High tech and healthcare firms do tend to have higher PE ratios, and manufacturing the least. The higher the market cap the higher the PE, and the lower the book-to-market ratio the higher the PE.

The takeaway is that payout and PE ratios move in opposite directions when we compare by broad industry categories, in the same direction when we examine by market cap (firm size), and in the opposite direction when we examine by book to market ratio (firm growth). Overall, a cursory examination of panels A of Figures 1 and 2 show that payout and PE ratios tend to move in opposite directions.

\section{FIGURE 1 DIVIDEND PAYOUT}

The top Panel shows the quarter-by-quarter average dividend payout ratio defined as average of quarterly cash dividend divided by quarterly net income, for all quarters from 1995 through 2016 where the recession quarters are 2001 Q2 - 2001 Q4 and 2008 Q1 - 2009 Q2 as determined by NBER, are shaded. The second panel shows the average payout ratio quarter-by-quarter for 5 industry groups. The third panel shows the average payout ratio quarter by quarter by three groups by firms' market capitalization. The last panel shows the average payout ratio quarter by quarter by three groups by firms' book-to-market value. All variables are defined in Appendix.

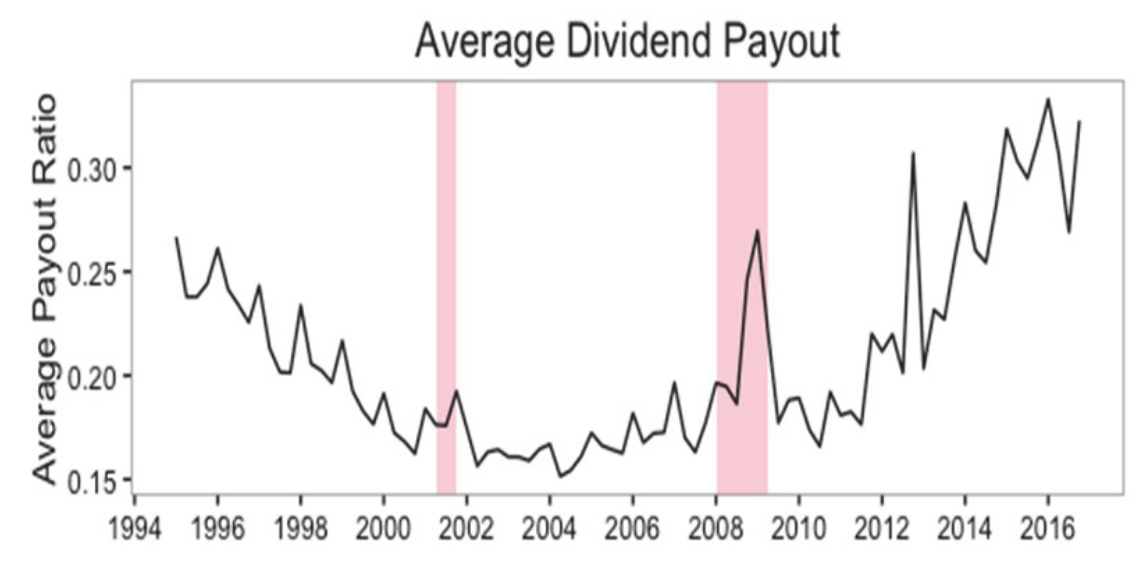

Journal of Accounting and Finance Vol. 20(2) 2020113 
Average Dividend Payout by Industries

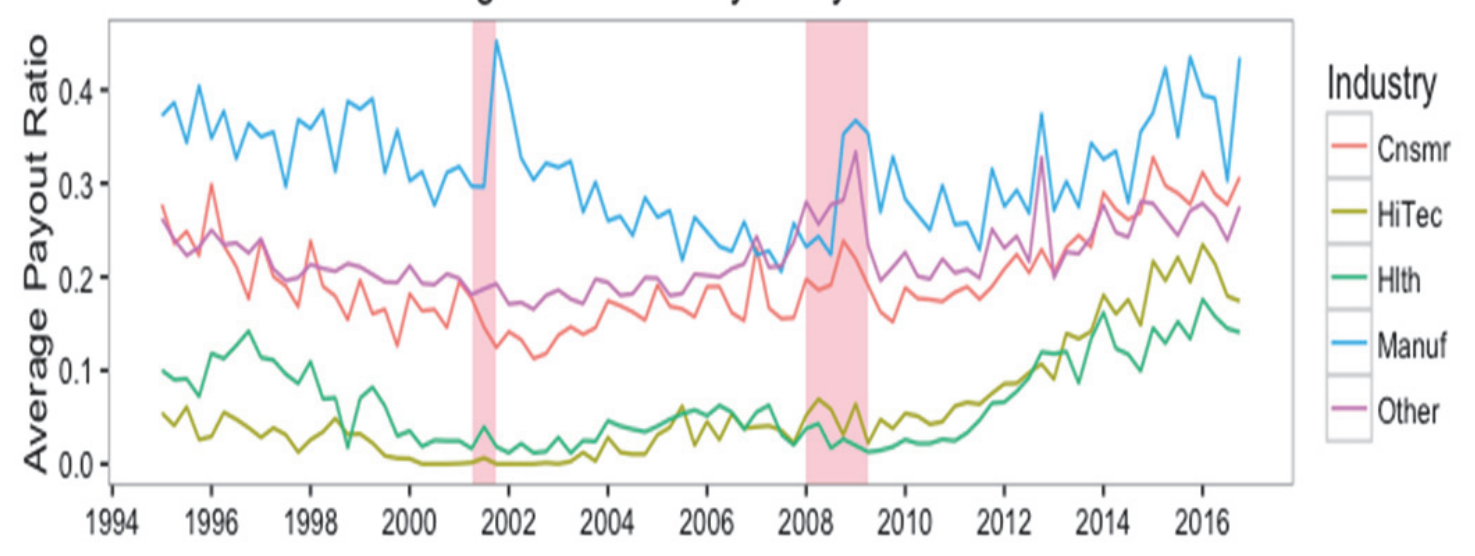

Average Dividend Payout by Market Cap

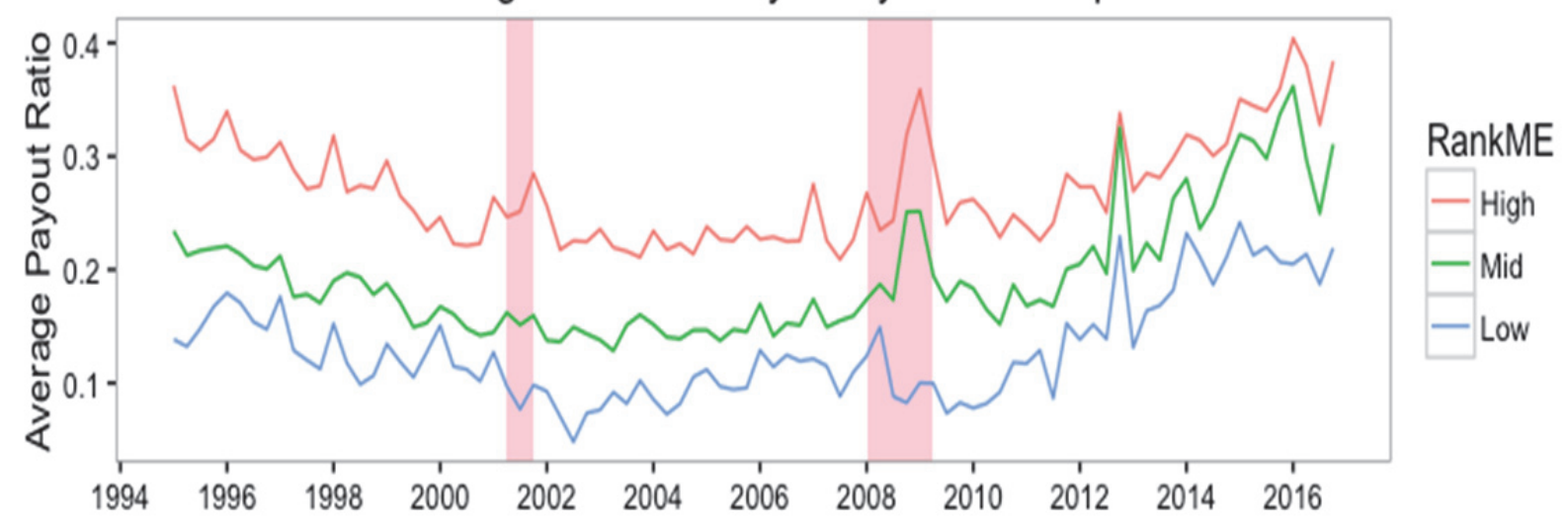

Average Dividend Payout by Book to Market Ratio

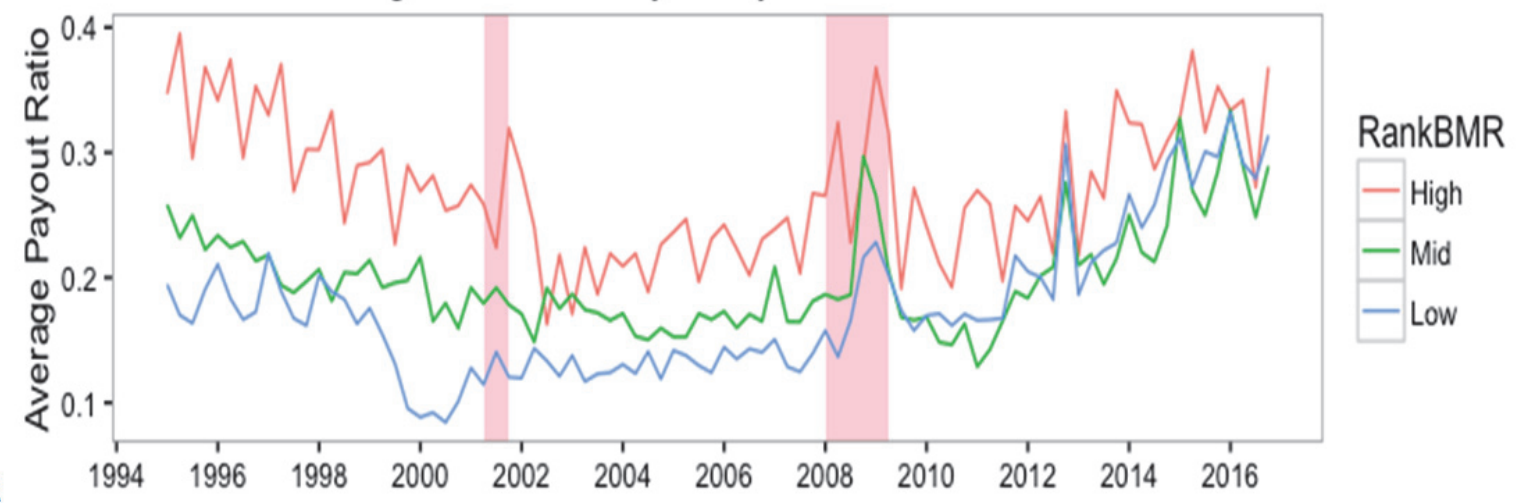




\section{FIGURE 2}

\section{PE RATIO}

The top Panel shows the quarter-by-quarter average PE ratio defined as average of quarterly stock price divided by quarterly EPS for all quarters from 1995 through 2016 where the recession quarters are 2001 Q2 - 2001 Q4 and 2008 Q1 - 2009 Q2 as determined by NBER, are shaded. The second panel shows the average payout ratio quarter-by-quarter for 5 industry groups. The third panel shows the average payout ratio quarter by quarter by three groups by firms' market capitalization. The last panel shows the average payout ratio quarter by quarter by three groups by firms' book to market value. All variables are defined in Appendix.

\section{Average P/E Ratio}

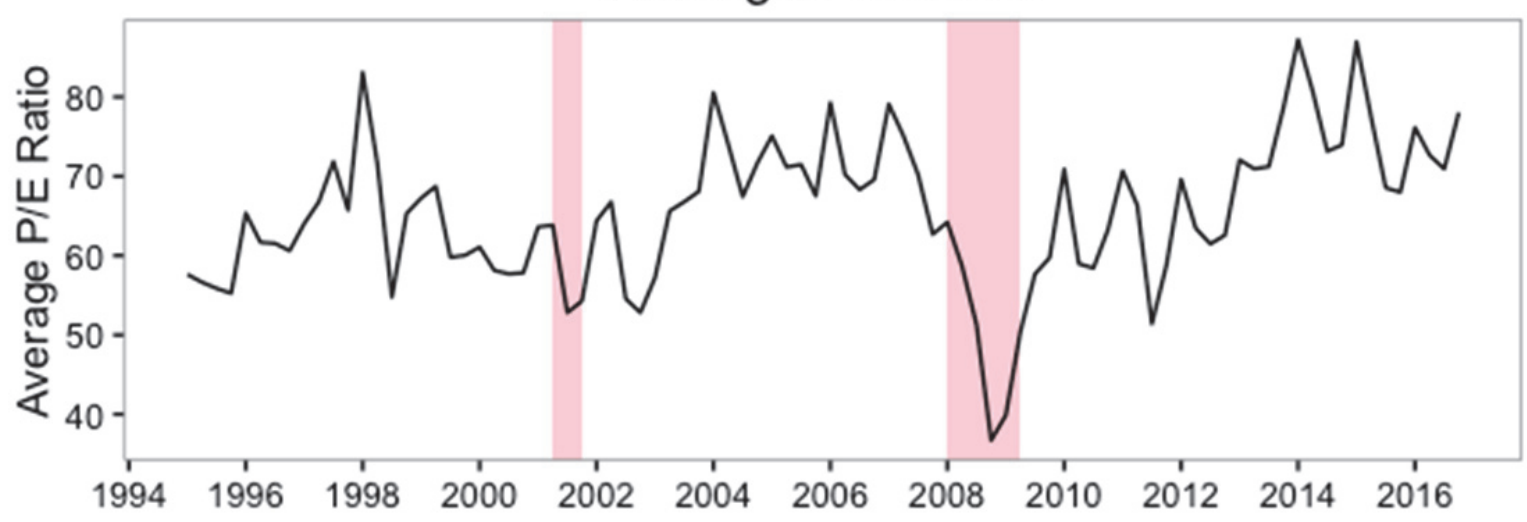

Average P/E by Industries

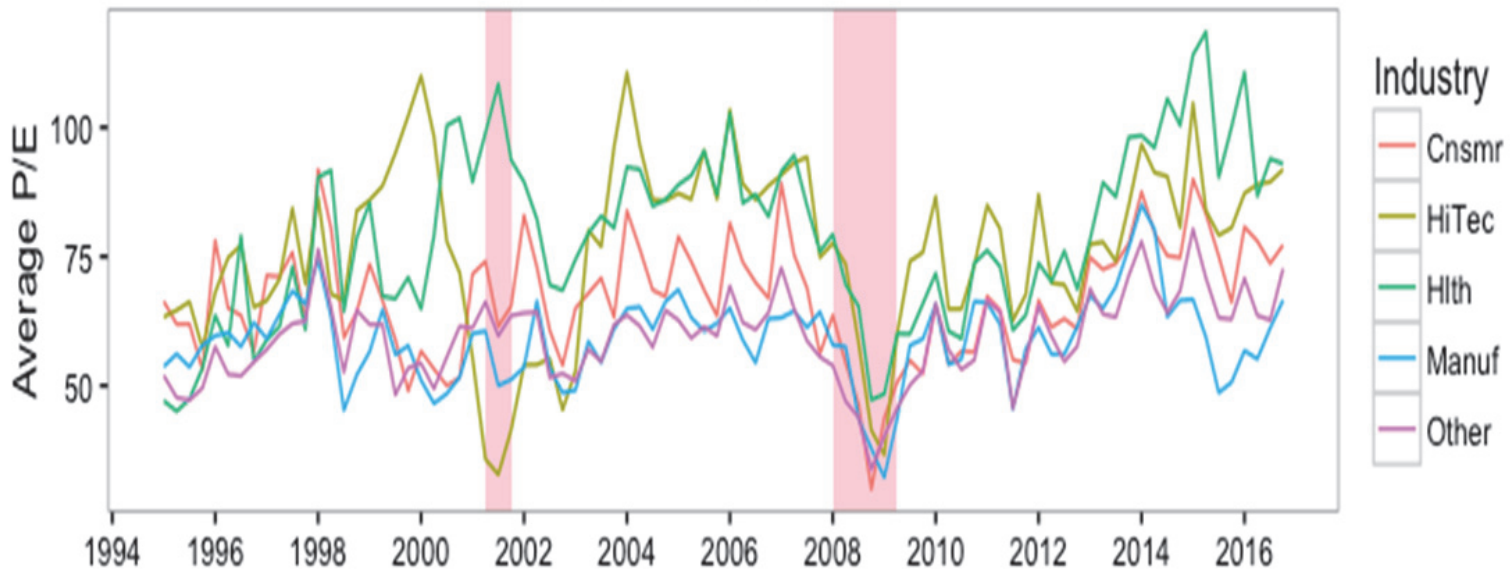




\section{Average P/E by Market Cap}

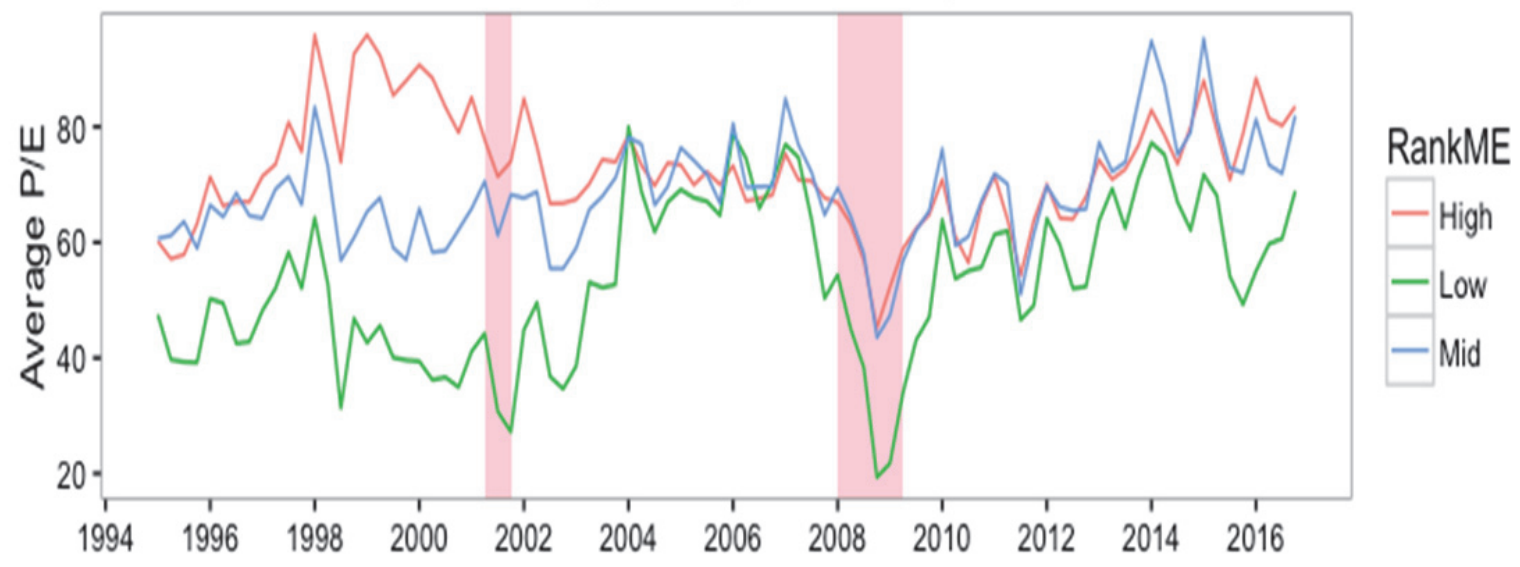

Average P/E by Book to Market Ratio

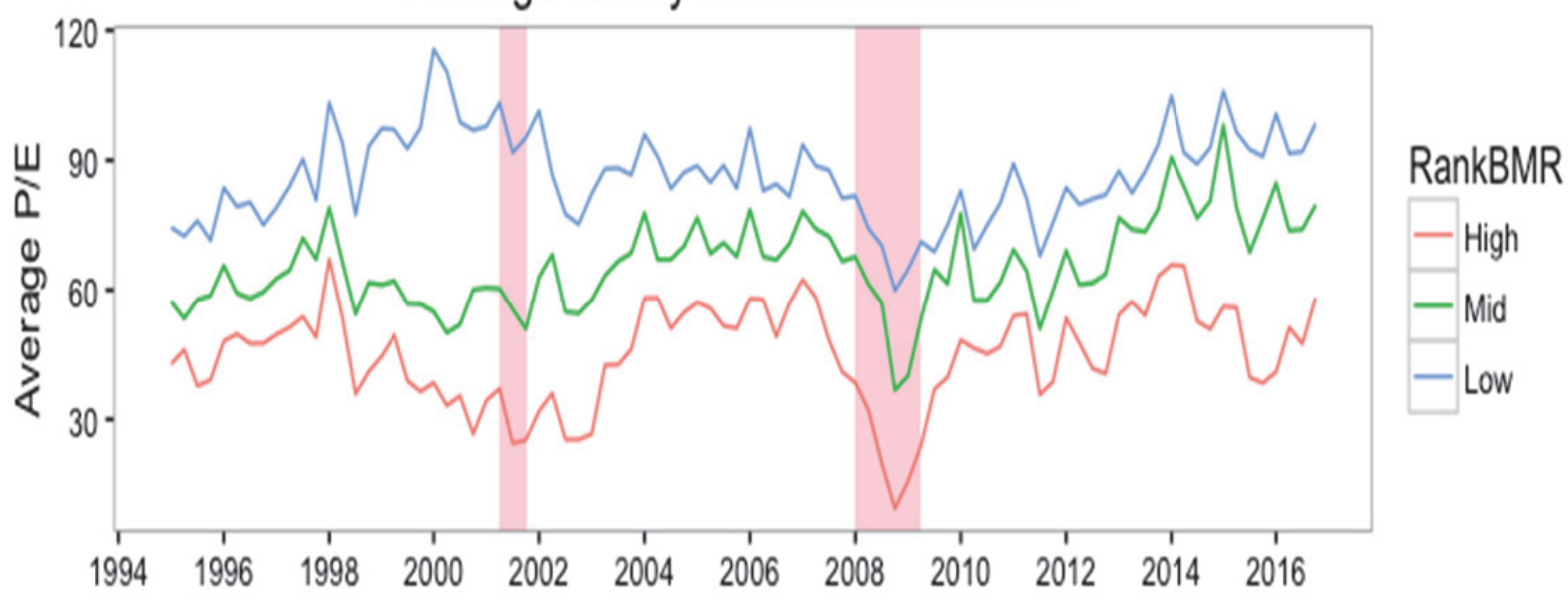




\section{TABLE 1 \\ DESCRIPTIVE STATISTICS: DIVIDEND PAYOUT RATIO}

The top panel shows the differences in mean Dividend Payout ratios for 5 Fama-French industry groups - Manufacturing, Consumer, Health, Hi-Tech, and Other. The middle panel shows the differences in mean Payout ratios for three groups of market capitalization (Market Value of Equity, MVE). The bottom panel shows the differences in mean Payout ratios for three groups of book-to-market (BM) value. All variables are defined in Appendix.

Average Dividend Payout by Industries

\begin{tabular}{cccccc}
\hline Mean $(\mathrm{X})$ & Mean(Y) & Mean(X-Y) & t-stat & df & p-value \\
\hline Manuf $=0.31$ & Other $=0.22$ & 0.09 & 40.4 & 50337 & $<2.2 \mathrm{e}-16$ \\
Other $=0.22$ & Cnsmr $=0.20$ & 0.02 & 11.6 & 48357 & $<2.2 \mathrm{e}-16$ \\
Cnsmr $=0.20$ & Hlth $=0.06$ & 0.14 & 71.4 & 25381 & $<2.2 \mathrm{e}-16$ \\
Hlth $=0.06$ & HiTec $=0.06$ & 0.00 & 0.12 & 13760 & 0.90 \\
\hline
\end{tabular}

Average dividend payout by Market Cap (MVE)

\begin{tabular}{cccccc}
\hline Mean $(\mathrm{X})$ & Mean(Y) & Mean(X-Y) & t-stat & df & p-value \\
\hline $\begin{array}{c}\text { High MVE }= \\
0.27\end{array}$ & $\begin{array}{c}\text { Mid MVE }= \\
0.19\end{array}$ & 0.08 & 45.7 & 72370 & $<2.2 \mathrm{e}-16$ \\
$\begin{array}{c}\text { Mid MVE }= \\
0.19\end{array}$ & $\begin{array}{c}\text { Low MVE }= \\
0.13\end{array}$ & 0.06 & 34.8 & 34.771 & $<2.2 \mathrm{e}-16$ \\
\hline
\end{tabular}

Average dividend payout by Book-to-Market (BM)

\begin{tabular}{cccccc}
\hline Mean $(\mathrm{X})$ & Mean(Y) & Mean(X-Y) & t-stat & df & p-value \\
\hline High BM $=0.27$ & $\begin{array}{c}\text { Mid BM }= \\
0.19\end{array}$ & 0.08 & 36.6 & 56125 & $<2.2 \mathrm{e}-16$ \\
$\begin{array}{c}\text { Mid BM }= \\
0.19\end{array}$ & $\begin{array}{c}\text { Low BM }= \\
0.17\end{array}$ & 0.02 & 12.7 & 71775 & $<2.2 \mathrm{e}-16$ \\
\hline
\end{tabular}


TABLE 2

DESCRIPTIVE STATISTICS: PE RATIO

The top panel shows the differences in mean Price-to-Earnings (PE) ratios for 5 Fama-French industry groups - Manufacturing, Consumer, Health, Hi-Tech, and Other. The middle panel shows the differences in mean PE ratios for three groups of market capitalization (Market Value of Equity, MVE). The bottom panel shows the differences in mean PE ratios for three groups of book-to-market (BM) value. All variables are defined in Appendix.

Average PE by Industry

\begin{tabular}{cccccc}
\hline Mean $(\mathrm{X})$ & Mean $(\mathrm{Y})$ & Mean $(\mathrm{X}-\mathrm{Y})$ & t-stat & df & p-value \\
\hline Hlth $=78.03$ & HiTec $=76.13$ & 1.90 & 2.51 & 20282 & 0.01 \\
HiTec $=76.13$ & Cnsmr $=66.22$ & 9.91 & 17.34 & 30784 & $<2.2 \mathrm{e}-16$ \\
Cnsmr $=66.22$ & Other $=59.80$ & 6.42 & 19.11 & 43835 & $<2.2 \mathrm{e}-16$ \\
Other $=59.80$ & Manuf $=59.38$ & 0.42 & 1.41 & 54063 & 0.16 \\
\hline
\end{tabular}

Average PE by Market Cap (MVE)

\begin{tabular}{cccccc}
\hline Mean $(\mathrm{X})$ & Mean $(\mathrm{Y})$ & Mean $(\mathrm{X}-\mathrm{Y})$ & t-stat & df & p-value \\
\hline $\begin{array}{c}\text { High MVE }= \\
71.91\end{array}$ & Mid MVE $=68.25$ & 3.66 & 12.75 & 72783 & $<2.2 \mathrm{e}-16$ \\
Mid MVE $=68.25$ & Low MVE $=52.41$ & 15.84 & 34.77 & 34.771 & $<2.2 \mathrm{e}-16$ \\
\hline
\end{tabular}

Average PE by Book to Market Ratio (BM)

\begin{tabular}{cccccc}
\hline Mean $(\mathrm{X})$ & Mean $(\mathrm{Y})$ & Mean $(\mathrm{X}-\mathrm{Y})$ & t-stat & $\mathrm{df}$ & $\mathrm{p}$-value \\
\hline $\begin{array}{c}\text { High BM }= \\
44.47\end{array}$ & $\begin{array}{c}\text { Mid BM }= \\
65.65\end{array}$ & -21.19 & -71.15 & 67201 & $<2.2 \mathrm{e}-16$ \\
$\begin{array}{c}\text { Mid BM }= \\
65.65\end{array}$ & $\begin{array}{c}\text { Low BM }= \\
85.40\end{array}$ & -19.75 & -68.65 & 69341 & $<2.2 \mathrm{e}-16$ \\
\hline
\end{tabular}

\section{MULTIVARIATE RESULTS}

Table 3 examines the determinants of the PE ratio, using the 2 variants of the following regression:

$$
\begin{aligned}
& \frac{P}{E} \text { Ratio }_{t+1}=\beta_{1} \times \text { Dividend Payout }_{t}+\beta_{2} \times \text { Recession }_{t}+\beta_{3} \times \text { Volatility }_{t}+
\end{aligned}
$$

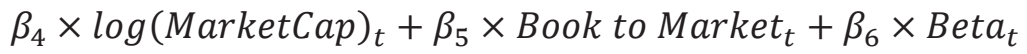

$$
\begin{aligned}
& +\beta_{7} \times \text { Industry }_{t}+\beta_{8} \times \text { Year }_{t}+\varepsilon
\end{aligned}
$$


where Book to Market $_{t}$ is a proxy for the inverse of growth options and $B e t a_{t}$ is calculated by running rolling-window regressions between stock returns and market return. We also control for year and industry fixed effects. In specification 1, we use price and EPS as of time $(\mathrm{t}+1)$, and in specification 2, we use price as of time $(\mathrm{t}+1)$ and EPS as of time $\mathrm{t}$.

We find that current period dividend payout is positively and significantly related to next period PE (at 1\% significance level) after controlling for year and industry fixed effects and firm beta. Contrary to univariate results, PE ratio is significantly lower for higher market cap firms, after controlling for other determinants. In line with the univariate results, PE ratio is significantly lower for higher book to market firms, because of lower perceived growth opportunities. These results are significant whether we use current earnings or trailing earnings. To examine this further, as well as to sort out the seeming conflict between univariate and multivariate results documented above, we run several regressions over groups of firms based on industry, market cap, book to market, and time period.

When we run regressions by groups divided by industry, market cap, book to market ratio and recession years, we find that results are more complicated. Table 4 reports regression results run separately over 90 groups overall, made up of 5 industry groups, 3 groups of market capitalization, 3 groups of book to market values, and whether or not it is a recession period. Only the specifications for which payout ratio is significant in explaining future $\mathrm{PE}$ at $5 \%$ or $1 \%$ levels are shown. Panel A shows the significant negative relations, and Panel B the significant positive relations.

For Consumer industry and High Technology industry, the correlation between payout and PE tends to be significantly negative, while it is significantly positive for Manufacturing and Other industries. In general, the positive relation between payout and future PE is for high market cap firms. There are no discernable relations for when the relation between payout and $\mathrm{PE}$ is positive or negative with regard to book-to-market and recession or expansion years, although in most of the regressions, book to market ratio has a significantly negative correlation with future PE.

The results are somewhat similar when we use trailing earnings instead of current earnings, in Table 5. For Consumer and High Tech industries, the correlation between payout and future PE tends to be significantly negative, while it is significantly positive for Manufacturing and Other industries. In general, we find positive relationship between payout and future PE for high market cap firms and for low bookto-market firms. 
TABLE 3

\section{DETERMINANTS OF PE RATIO}

This table reports the regression coefficients and the associated heteroscedasticity consistent t-statistics (along with adjusted $\mathrm{R}^{2}$ ) of 2 specifications of the determinants of $\mathrm{P} / \mathrm{E}$ ratio, where the dividend payout ratio is the key explanatory variable:

$P /$ Ratio $_{t+1}=\beta_{1} \times$ Dividend Payout $_{t}+\beta_{2} \times$ Recession $_{t}+\beta_{3} \times$ Volatility $_{t}+\beta_{4} \times{\text { Log } \text { Market }_{\text {Cap }}}_{t}$

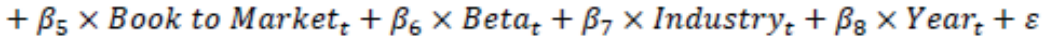

All variables are defined in Appendix.

\begin{tabular}{|c|c|c|}
\hline & $\mathrm{P} /$ E Ratio $_{\mathrm{t}+1}$ & $\begin{array}{c}\mathrm{P} / \mathrm{E} \mathrm{Ratio}_{\mathrm{t}+1} \\
\text { (trailing earnings) }\end{array}$ \\
\hline \multirow{2}{*}{ Dividend Payout $_{t}$} & $2.74 * * *$ & $4.72 * * *$ \\
\hline & $(4.46)$ & (8.69) \\
\hline \multirow{2}{*}{ Recession } & -1.382 & $-4.05 * * *$ \\
\hline & $(-1.43)$ & $(-4.73)$ \\
\hline \multirow{2}{*}{ Volatility } & -22.74 & 38.96. \\
\hline & $(-0.93)$ & $(1.79)$ \\
\hline \multirow{2}{*}{ Log(Market Cap) } & $-0.48 * * *$ & $-1.04 * * *$ \\
\hline & $(-4.35)$ & $(-10.70)$ \\
\hline \multirow{2}{*}{ Book to Market } & $-34.43 * * *$ & $-35.66 * * *$ \\
\hline & $(-70.80)$ & $(-83.03)$ \\
\hline \multirow{2}{*}{ Beta } & $3.69 * * *$ & $4.14 * * *$ \\
\hline & $(7.95)$ & $(10.03)$ \\
\hline Industry Fixed Effects & Yes & Yes \\
\hline Year Fixed Effects & Yes & Yes \\
\hline Adjusted $\mathrm{R}^{2}$ & 0.78 & 0.84 \\
\hline
\end{tabular}


TABLE 4

DETERMINANTS OF PE RATIO BY GROUPS USING CONCURRENT EARNINGS

This table reports regression coefficients and the associated heteroscedasticity consistent t-statistics (along with adjusted $\mathrm{R}^{2}$ ) of the determinants of $\mathrm{P} / \mathrm{E}$ ratio, where the dividend payout ratio is the key explanatory variable:

$$
\begin{aligned}
P / \text { Ratio }_{t+1}= & \beta_{0}+\beta_{1} \times \text { Dividend Payout }_{t}+\beta_{2} \times \text { Volatility }_{t}+\beta_{3} \times \text { Log Market Cap }_{t}+\beta_{4} \times{\text { Book } \text { to Market }_{t}+\beta_{5}} \times \text { Beta }_{t}+\varepsilon
\end{aligned}
$$

The earnings here are concurrent earnings (to price). These regressions are run separately over 90 groups overall, made up of 5 industry groups, 3 groups of market capitalization, 3 groups of book to market values, and whether it is a recession period or not. Only the specifications for which Dividend Payout ratio is significant at $5 \%$ or $1 \%$ levels are shown. Panel A shows significant negative relations, and Panel

\begin{tabular}{|c|c|c|c|c|c|c|c|c|c|}
\hline Industry & $\begin{array}{l}\text { Rank } \\
\text { MVE }\end{array}$ & $\begin{array}{c}\text { Rank } \\
\text { BM }\end{array}$ & REC & $\begin{array}{c}\text { Dividend } \\
\text { Payout }\end{array}$ & Volatility & $\begin{array}{c}\text { LogMarket } \\
\text { Cap }\end{array}$ & $\begin{array}{l}\text { Book to } \\
\text { Market }\end{array}$ & Beta & Adjusted $\mathrm{R}^{2}$ \\
\hline Cnsmr & High & Low & 0 & $\begin{array}{c}-11.0 * * * \\
(-4.4)\end{array}$ & $\begin{array}{c}429.9 * * * \\
(4.6)\end{array}$ & $\begin{array}{c}2.2 * * * \\
(3.3)\end{array}$ & $\begin{array}{c}-55.3 * * * \\
(-10.1)\end{array}$ & $\begin{array}{c}9.4 * * * \\
(5.0)\end{array}$ & 0.06 \\
\hline Cnsmr & Mid & Low & 0 & $\begin{array}{c}-21.2 * * * \\
(-5.5)\end{array}$ & $\begin{array}{l}-44.9 \\
(-0.3)\end{array}$ & $\begin{array}{c}6.2 * * * \\
(3.7)\end{array}$ & $\begin{array}{c}-40.6 * * * \\
(-5.2)\end{array}$ & $\begin{array}{c}1.7 \\
(0.7)\end{array}$ & 0.03 \\
\hline HiTec & High & High & 0 & $\begin{array}{c}-49.7 * * * \\
(-5.5)\end{array}$ & $\begin{array}{l}-272.1 \\
(-0.9)\end{array}$ & $\begin{array}{c}8.6 * * * \\
(4.1)\end{array}$ & $\begin{array}{l}-14.9 \\
(-1.4)\end{array}$ & $\begin{array}{c}6.1 \\
(0.8)\end{array}$ & 0.10 \\
\hline HiTec & High & Low & 0 & $\begin{array}{c}-17.2 * * * \\
(-4.8)\end{array}$ & $\begin{array}{c}376.1 * * * \\
(3.8)\end{array}$ & $\begin{array}{l}-1.0 \\
(-1.3)\end{array}$ & $\begin{array}{c}-34.5 * * * \\
(-4.3)\end{array}$ & $\begin{array}{c}13.1 * * * \\
(5.3)\end{array}$ & 0.07 \\
\hline HiTec & High & Mid & 0 & $\begin{array}{c}-24.5 * * * \\
(-4.5)\end{array}$ & $\begin{array}{c}-298.7 * \\
(-1.8)\end{array}$ & $\begin{array}{c}0.6 \\
(0.5)\end{array}$ & $\begin{array}{c}-84.4 * * * \\
(-6.9)\end{array}$ & $\begin{array}{l}6.09 \\
(1.5)\end{array}$ & 0.07 \\
\hline Other & High & Low & 0 & $\begin{array}{c}-10.9 * * * \\
(-3.5)\end{array}$ & $\begin{array}{l}13.1 \\
(0.1)\end{array}$ & $\begin{array}{l}-1.7 * \\
(-2.4)\end{array}$ & $\begin{array}{c}-88.4 * * * \\
(-14.0)\end{array}$ & $\begin{array}{c}5.7 * * \\
(2.8)\end{array}$ & 0.09 \\
\hline
\end{tabular}
$\mathrm{B}$ significant positive relations. All variables are defined in Appendix. 
Panel B

\begin{tabular}{|c|c|c|c|c|c|c|c|c|c|}
\hline Industry & $\begin{array}{l}\text { Rank } \\
\text { MVE }\end{array}$ & $\begin{array}{c}\text { Rank } \\
\text { BM }\end{array}$ & REC & $\begin{array}{c}\text { Dividend } \\
\text { Payout }\end{array}$ & Volatility & $\begin{array}{c}\text { LogMarket } \\
\text { Cap }\end{array}$ & $\begin{array}{l}\text { Book to } \\
\text { Market }\end{array}$ & Beta & Adjusted $\mathrm{R}^{2}$ \\
\hline Manuf & High & Mid & 0 & $\begin{array}{c}21.3 * * * \\
(6.2)\end{array}$ & $\begin{array}{l}77.7 \\
(0.6)\end{array}$ & $\begin{array}{l}-1.8 * \\
(-2.0)\end{array}$ & $\begin{array}{c}-35.3 * * * \\
(-4.6)\end{array}$ & $\begin{array}{c}0.3 \\
(0.1)\end{array}$ & 0.02 \\
\hline Manuf & High & Mid & 1 & $\begin{array}{c}35.0 * * \\
(2.7)\end{array}$ & $\begin{array}{l}639.8 \\
(1.5)\end{array}$ & $\begin{array}{l}-7.8 * \\
(-1.9)\end{array}$ & $\begin{array}{l}-12.2 \\
(-0.4)\end{array}$ & $\begin{array}{c}-18.8 * \\
(-1.9)\end{array}$ & 0.04 \\
\hline Manuf & Mid & Low & 0 & $\begin{array}{c}11.7 * * \\
(3.0)\end{array}$ & $\begin{array}{l}-85.7 \\
(-0.6)\end{array}$ & $\begin{array}{c}4.3 * * \\
(2.7)\end{array}$ & $\begin{array}{c}33.5 * * * \\
(4.4)\end{array}$ & $\begin{array}{c}2.8 \\
(1.3)\end{array}$ & 0.03 \\
\hline Manuf & Mid & Mid & 0 & $\begin{array}{l}7.4 * * \\
(2.5)\end{array}$ & $\begin{array}{l}-67.7 \\
(-0.7)\end{array}$ & $\begin{array}{c}4.0 * * \\
(3.1)\end{array}$ & $\begin{array}{c}-24.2 * * * \\
(-3.8)\end{array}$ & $\begin{array}{l}-0.1 \\
(-0.1)\end{array}$ & 0.01 \\
\hline Other & High & High & 0 & $\begin{array}{c}12.8 * * * \\
(4.6)\end{array}$ & $\begin{array}{c}221.9 * \\
(2.4)\end{array}$ & $\begin{array}{l}1.4 * \\
(2.1)\end{array}$ & $\begin{array}{c}-7.8 * * \\
(-3.0)\end{array}$ & $\begin{array}{c}-5.0 * * \\
(-2.6)\end{array}$ & 0.02 \\
\hline Other & Low & High & 0 & $\begin{array}{c}10.0 * * * \\
(3.6)\end{array}$ & $\begin{array}{c}-257.2 * * \\
(-2.7)\end{array}$ & $\begin{array}{c}1.8 \\
(1.3)\end{array}$ & $\begin{array}{c}-26.4 * * * \\
(-8.6)\end{array}$ & $\begin{array}{c}8.3 * * * \\
(5.0)\end{array}$ & 0.06 \\
\hline Other & Low & Low & 0 & $\begin{array}{c}19.4 * * * \\
(3.9)\end{array}$ & $\begin{array}{l}100.2 \\
(0.5)\end{array}$ & $\begin{array}{c}14.2 * * * \\
(5.0)\end{array}$ & $\begin{array}{c}16.7 * \\
(1.6)\end{array}$ & $\begin{array}{l}-3.3 \\
(-1.1)\end{array}$ & 0.04 \\
\hline Other & Mid & High & 0 & $\begin{array}{c}15.1 * * * \\
(6.0)\end{array}$ & $\begin{array}{c}148.0 * \\
(1.8)\end{array}$ & $\begin{array}{c}5.6 * * * \\
(4.9)\end{array}$ & $\begin{array}{c}-15.0 * * * \\
(-5.6)\end{array}$ & $\begin{array}{c}2.6 \\
(1.6)\end{array}$ & 0.03 \\
\hline
\end{tabular}

$*, * *$, and $* * *$ denote significant at the $10 \%, 5 \%$ and $1 \%$ level respectively.

TABLE 5

DETERMINANTS OF PE RATIO BY GROUPS: USING TRAILING EARNINGS

This table reports regression coefficients and the associated heteroscedasticity consistent t-statistics (along with adjusted $\mathrm{R}^{2}$ ) of the determinants of $\mathrm{P} / \mathrm{E}$ ratio, where the dividend payout ratio is the key explanatory variable:

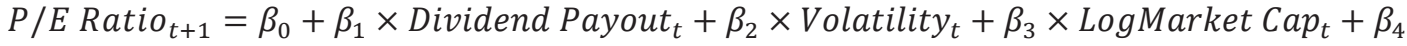

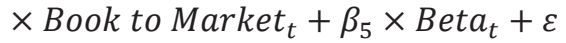

The earnings here are trailing earnings (compared to price). These regressions are run separately over 90 groups overall, made up of 5 industry groups, 3 groups of market capitalization, 3 groups of book to market values, and whether it is a recession period or not. Only the specifications for which Dividend Payout ratio is significant at $5 \%$ or $1 \%$ levels are shown. Panel A shows significant negative relations, and Panel B significant positive relations. All variables are defined in Appendix. 
Panel A

\begin{tabular}{|c|c|c|c|c|c|c|c|c|c|}
\hline Industry & $\begin{array}{l}\text { Rank } \\
\text { MVE }\end{array}$ & $\begin{array}{c}\text { Rank } \\
\text { BM }\end{array}$ & REC & $\begin{array}{c}\text { Dividend } \\
\text { Payout }\end{array}$ & Volatility & $\begin{array}{l}\text { LogMarket } \\
\text { Cap }\end{array}$ & $\begin{array}{l}\text { Book to } \\
\text { Market }\end{array}$ & Beta & Adjusted $\mathrm{R}^{2}$ \\
\hline Cnsmr & High & Low & 0 & $\begin{array}{c}-7.4 * * * \\
(-3.5)\end{array}$ & $\begin{array}{c}503.6 * * * \\
(6.4)\end{array}$ & $\begin{array}{c}0.8 \\
(1.5)\end{array}$ & $\begin{array}{c}-60.4 * * * \\
(-12.9)\end{array}$ & $\begin{array}{c}9.2 * * * \\
(5.7)\end{array}$ & 0.08 \\
\hline Cnsmr & High & Low & 1 & $\begin{array}{c}-32.8 * * * \\
(-5.0)\end{array}$ & $\begin{array}{c}547.4 * \\
(2.2)\end{array}$ & $\begin{array}{c}6.5 * * \\
(2.9)\end{array}$ & $\begin{array}{c}-24.9 * \\
(-1.9)\end{array}$ & $\begin{array}{c}-18.4 * * * \\
(-3.3)\end{array}$ & 0.10 \\
\hline Cnsmr & Mid & Low & 0 & $\begin{array}{c}-15.2 * * * \\
(-4.8)\end{array}$ & $\begin{array}{c}162.0 * \\
(1.7)\end{array}$ & $\begin{array}{c}1.6 \\
(1.1)\end{array}$ & $\begin{array}{c}-55.3 * * * \\
(-8.4)\end{array}$ & $\begin{array}{c}0.9 \\
(0.5)\end{array}$ & 0.05 \\
\hline HiTec & High & Low & 0 & $\begin{array}{c}-16.9 * * * \\
(-5.0)\end{array}$ & $\begin{array}{c}854.9 * * * \\
(9.0)\end{array}$ & $\begin{array}{c}-0.4 \\
(-0.6)\end{array}$ & $\begin{array}{c}-36.9 * * * \\
(-4.9)\end{array}$ & $\begin{array}{c}12.7 * * * \\
(5.4)\end{array}$ & 0.13 \\
\hline HiTec & High & Low & 1 & $\begin{array}{c}-30.1 * * \\
(-2.9)\end{array}$ & $\begin{array}{c}1107.8 * \\
(2.4)\end{array}$ & $\begin{array}{l}8.7 * \\
(2.5)\end{array}$ & $\begin{array}{l}-17.4 \\
(-0.7)\end{array}$ & $\begin{array}{l}-8.1 \\
(-0.6)\end{array}$ & 0.06 \\
\hline HiTec & High & Mid & 0 & $\begin{array}{c}-20.0 * * * \\
(-3.7)\end{array}$ & $\begin{array}{l}-67.1 \\
(-0.4)\end{array}$ & $\begin{array}{c}0.3 \\
(0.2)\end{array}$ & $\begin{array}{c}-100.1 * * * \\
(-8.5)\end{array}$ & $\begin{array}{c}4.8 \\
(1.2)\end{array}$ & 0.08 \\
\hline Hlth & High & Low & 1 & $\begin{array}{c}-47.5 * * \\
(-3.2)\end{array}$ & $\begin{array}{c}1404.6^{* * *} \\
(3.7)\end{array}$ & $\begin{array}{c}1.6 \\
(0.5)\end{array}$ & $\begin{array}{c}-211.6 * * * \\
(-8.4)\end{array}$ & $\begin{array}{c}-36.1 * * * \\
(-3.3)\end{array}$ & 0.32 \\
\hline Hlth & Mid & Low & 0 & $\begin{array}{c}-24.3 * * * \\
(-3.4)\end{array}$ & $\begin{array}{c}-482.6 * * * \\
(-3.3)\end{array}$ & $\begin{array}{l}5.1 * \\
(2.1)\end{array}$ & $\begin{array}{c}-149.2 * * * \\
(-10.0)\end{array}$ & $\begin{array}{c}2.8 \\
(0.7)\end{array}$ & 0.13 \\
\hline
\end{tabular}

\begin{tabular}{|c|c|c|c|c|c|c|c|c|c|}
\hline Industry & $\begin{array}{l}\text { Rank } \\
\text { MVE }\end{array}$ & $\begin{array}{c}\text { Rank } \\
\text { BM }\end{array}$ & REC & $\begin{array}{c}\text { Dividend } \\
\text { Payout }\end{array}$ & Volatility & $\begin{array}{c}\text { LogMarket } \\
\text { Cap }\end{array}$ & $\begin{array}{l}\text { Book to } \\
\text { Market }\end{array}$ & Beta & Adjusted $\mathrm{R}^{2}$ \\
\hline Cnsmr & High & High & 0 & $\begin{array}{c}16.2 * * \\
(3.0)\end{array}$ & $\begin{array}{l}261.4 \\
(1.3)\end{array}$ & $\begin{array}{c}-1.4 \\
(-0.9)\end{array}$ & $\begin{array}{c}-27.6 * * * \\
(-4.1)\end{array}$ & $\begin{array}{c}-16.6 * * * \\
(-4.1)\end{array}$ & 0.07 \\
\hline Cnsmr & High & High & 1 & $\begin{array}{c}143.0 * * * \\
(4.2)\end{array}$ & $\begin{array}{l}454.7 \\
(0.3)\end{array}$ & $\begin{array}{c}5.2 \\
(0.4)\end{array}$ & $\begin{array}{l}-69.7 \\
(-1.5)\end{array}$ & $\begin{array}{l}-39.0 \\
(-1.4)\end{array}$ & 0.30 \\
\hline Cnsmr & Mid & Mid & 0 & $\begin{array}{c}8.5 * * * \\
(3.5)\end{array}$ & $\begin{array}{c}-368.6 * * * \\
(-4.7)\end{array}$ & $\begin{array}{c}0.6 \\
(0.5)\end{array}$ & $\begin{array}{c}-37.8 * * * \\
(-6.9)\end{array}$ & $\begin{array}{c}6.1 * * * \\
(4.0)\end{array}$ & 0.05 \\
\hline Hlth & Mid & Mid & 0 & $\begin{array}{c}20.8 * * \\
(2.6)\end{array}$ & $\begin{array}{c}-279.3 * \\
(-1.8)\end{array}$ & $\begin{array}{c}0.6 \\
(0.2)\end{array}$ & $\begin{array}{c}-77.0 * * * \\
(-6.2)\end{array}$ & $\begin{array}{c}1.0 \\
(0.27)\end{array}$ & 0.07 \\
\hline Manuf & High & High & 0 & $\begin{array}{c}23.8 * * * \\
(9.5)\end{array}$ & $\begin{array}{l}95.3 \\
(0.8)\end{array}$ & $\begin{array}{l}-0.1 \\
(-0.1)\end{array}$ & $\begin{array}{c}-25.6 * * * \\
(-8.8)\end{array}$ & $\begin{array}{c}1.7 \\
(0.9)\end{array}$ & 0.07 \\
\hline Manuf & High & Low & 1 & $\begin{array}{c}38.1 * * * \\
(4.5)\end{array}$ & $\begin{array}{c}356.8 * \\
(1.6)\end{array}$ & $\begin{array}{c}0.2 \\
(0.1)\end{array}$ & $\begin{array}{c}-34.0 * \\
(-2.0)\end{array}$ & $\begin{array}{c}-24.1 * * * \\
(-3.7)\end{array}$ & 0.15 \\
\hline Manuf & High & Mid & 0 & $\begin{array}{c}24.0 * * * \\
(7.5)\end{array}$ & $\begin{array}{c}235.8 * \\
(2.1)\end{array}$ & $\begin{array}{c}-2.7 * * * \\
(-3.3)\end{array}$ & $\begin{array}{c}-45.4 * * * \\
(-6.4)\end{array}$ & $\begin{array}{c}1.3 \\
(0.7)\end{array}$ & 0.04 \\
\hline Manuf & High & Mid & 1 & $\begin{array}{c}32.5 * * * \\
(4.2)\end{array}$ & $\begin{array}{c}534.9 * \\
(2.1)\end{array}$ & $\begin{array}{l}-3.0 \\
(-1.2)\end{array}$ & $\begin{array}{c}-49.6 * * \\
(-3.2)\end{array}$ & $\begin{array}{c}-8.8 \\
(-1.5)\end{array}$ & 0.07 \\
\hline Manuf & Mid & High & 0 & $\begin{array}{c}12.0 * * * \\
(5.3)\end{array}$ & $\begin{array}{c}-270.2 * * \\
(-3.1)\end{array}$ & $\begin{array}{c}5.5 * * * \\
(4.8)\end{array}$ & $\begin{array}{c}-18.7 * * * \\
(-6.9)\end{array}$ & $\begin{array}{c}6.1 * * * \\
(3.9)\end{array}$ & 0.06 \\
\hline
\end{tabular}




\begin{tabular}{|c|c|c|c|c|c|c|c|c|c|}
\hline Manuf & Mid & Mid & 1 & $\begin{array}{c}29.3 * * * \\
(3.9)\end{array}$ & $\begin{array}{l}113.6 \\
(0.5)\end{array}$ & $\begin{array}{c}-13.6 \text { ** } \\
(-2.8)\end{array}$ & $\begin{array}{c}-59.0 * * * \\
(-4.0)\end{array}$ & $\begin{array}{c}-4.1 \\
(-0.6)\end{array}$ & 0.08 \\
\hline Other & High & High & 0 & $\begin{array}{c}21.3 * * * \\
(8.1)\end{array}$ & $\begin{array}{c}337.9 * * * \\
(4.0)\end{array}$ & $\begin{array}{l}1.0 * \\
(1.6)\end{array}$ & $\begin{array}{c}-0.8 \\
(-0.3)\end{array}$ & $\begin{array}{c}-7.6 * * * \\
(-4.2)\end{array}$ & 0.04 \\
\hline Other & High & Mid & 0 & $\begin{array}{c}11.1 \text { *** } \\
(4.5)\end{array}$ & $\begin{array}{c}312.5 * * * \\
(4.3)\end{array}$ & $\begin{array}{c}-0.6 \\
(-1.1)\end{array}$ & $\begin{array}{c}-61.0 * * * \\
(-12.8)\end{array}$ & $\begin{array}{c}-4.7 * * \\
(-3.0)\end{array}$ & 0.06 \\
\hline Other & High & Mid & 1 & $\begin{array}{c}22.7 * * \\
(2.62)\end{array}$ & $\begin{array}{c}-446.3 * \\
(-1.8)\end{array}$ & $\begin{array}{c}-0.6 \\
(-0.2)\end{array}$ & $\begin{array}{c}-52.3 * * \\
(-3.1)\end{array}$ & $\begin{array}{l}3.1 \\
(0.4)\end{array}$ & 0.09 \\
\hline Other & Low & High & 0 & $\begin{array}{c}14.6^{* * *} \\
(5.8)\end{array}$ & $\begin{array}{c}-249.5 * * \\
(-2.9)\end{array}$ & $\begin{array}{c}-1.5 \\
(-1.2)\end{array}$ & $\begin{array}{c}-23.1 * * * \\
(-8.2)\end{array}$ & $\begin{array}{c}7.9 * * * \\
(5.3)\end{array}$ & 0.06 \\
\hline Other & Low & Low & 0 & $\begin{array}{c}20.4 * * * \\
(4.7)\end{array}$ & $\begin{array}{l}78.1 \\
(0.5)\end{array}$ & $\begin{array}{c}16.0 \text { *** } \\
(6.4)\end{array}$ & $\begin{array}{c}29.9^{* * *} \\
(3.4)\end{array}$ & $\begin{array}{l}-3.6 \\
(-1.3)\end{array}$ & 0.08 \\
\hline Other & Mid & High & 0 & $\begin{array}{c}22.3 * * * \\
(9.9)\end{array}$ & $\begin{array}{c}227.0 * * \\
(3.1)\end{array}$ & $\begin{array}{c}4.3 * * * \\
(4.2)\end{array}$ & $\begin{array}{c}-13.6 * * * \\
(-5.6)\end{array}$ & $\begin{array}{l}0.03 \\
(0.0)\end{array}$ & 0.05 \\
\hline
\end{tabular}

$*, * *$, and $* * *$ denote significant at the $10 \%, 5 \%$ and $1 \%$ level respectively.

To directly examine the impact of changes on changes, we regress changes in PE on changes in payout. We have seen that the correlation between payout and PE levels tend to be significantly positive for high market cap firms and manufacturing firms, and tends to be negative for high book to market firms. Therefore, market cap, book-to-market ratio, and whether a firm is in the manufacturing sector or not matters for determining PE levels as a function of payout levels. We control for this using the

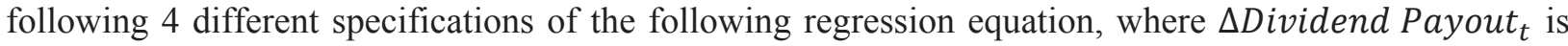
the difference between dividend payout at $\mathrm{t}$ and $\mathrm{t}-1$ and $\Delta P / E$ Ratio $_{t+1}$ is the difference in PE ratio between times $\mathrm{t}+1$ and $\mathrm{t}$.

$$
\begin{aligned}
& \Delta P / \text { Ratio }_{t+1}=\beta_{1} \times \Delta \text { Dividend Payout }_{t}+\beta_{2} \times \text { Recession }_{t}+\beta_{3} \times \text { Volatility }_{t}+\beta_{4} \\
& \times \text { LogMarket Cap }_{t}+\beta_{5} \times \text { Book to Market }_{t} \\
& +\beta_{6} \times \text { Beta }_{t}+\beta_{7} \times \Delta \text { Dividend Payout }_{t} \times \text { LogMarket Cap }_{t}+\beta_{8}
\end{aligned}
$$

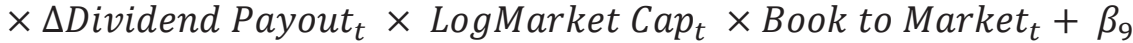

$$
\begin{aligned}
& \times \Delta \text { Dividend Payout }_{t} \times \text { LogMarket Cap }_{t} \times \text { Book to Market }_{t} \times \text { Manufacturing } \\
& +\beta_{10} \times \text { Industry }_{t}+\beta_{11} \times \text { Year }_{t}+\varepsilon \text {, }
\end{aligned}
$$

Table 6 shows that, irrespective of the specification used, next period PE change is significantly and negatively associated with current-period dividend-payout change. The previous results have shown that, on average, dividend payout level is significantly and positively related to PE level. But once the levels are determined, any change in payout negatively affects future changes in PE. As before, we run the regression specification separately over 90 groups, made up of 5 industry groups, 3 groups of market capitalization, 3 groups of book to market values, and whether or not it is a recession period. Only the specifications for which $\Delta$ Dividend Payout $t_{t}$ is significant at the $5 \%$ and $1 \%$ levels are shown in Table 7. We find that the above documented negative relation is robust: it not only in the overall sample, but also in almost every industry and firm type subsample.

Finally, we regress change in volatility in the next period, as defined by the standard deviation of stock returns, on change in payout, to check the impact of current-period payout changes on future risk, using the following regression specification: 


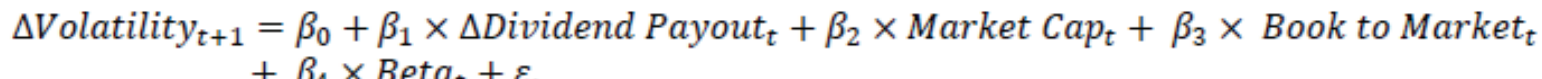

$$
+\beta_{4} \times \text { Beta }_{t}+\varepsilon
$$

and find that there is indeed a significant and positive relationship (Table 8). Thus increased payout in the current period signals increased risk in future (perhaps due to reduced growth opportunities) and, and hence the future PE decreases.

In summary, current period dividend payout change is significantly and negatively associated with future PE change likely because an increase in current period payout signals reduced investment opportunities which reduces future PE. Moreover, the relation between payout and PE levels and between payout changes and PE changes depends on industry, firm size, perceived growth opportunities (proxied by the book-to-market ratio), and other measures of firm risk.

\section{TABLE 6}

\section{DETERMINANTS OF CHANGES IN PE RATIO}

This table reports regression coefficients and the associated heteroscedasticity consistent t-statistics (along with adjusted $\mathrm{R}^{2}$ ) of 4 different specifications for explaining changes in $\mathrm{P} / \mathrm{E}$ ratio, where changes in dividend payout ratio is the key explanatory variable:

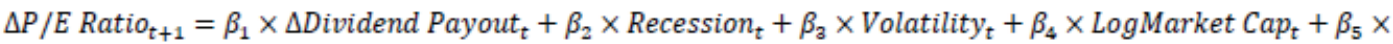

$$
\begin{aligned}
& \text { Book to Market } t+\beta_{6} \times \text { Beta }_{t}+\beta_{7} \times \Delta \text { Dividend Payout }_{t} \times \text { LogMarket Cap }_{t}+\beta_{8} \times
\end{aligned}
$$

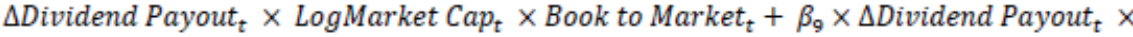

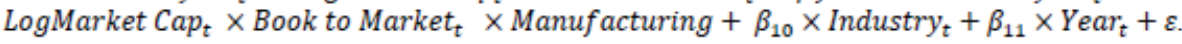

\begin{tabular}{|c|c|c|c|c|}
\hline & $\Delta P /$ Ratio $_{t+1}$ & $\Delta P /$ Ratio $_{t+1}$ & $\Delta P /$ Ratio $_{t+1}$ & $\Delta P / E$ Ratio $_{t+1}$ \\
\hline$\Delta$ Dividend Payout $_{t}$ & $\begin{array}{c}-69.91 * * * \\
(-20.81)\end{array}$ & $\begin{array}{c}-51.30 * * \\
(-2.74)\end{array}$ & $\begin{array}{c}-73.33 * * * \\
(-11.44)\end{array}$ & $\begin{array}{c}-51.48 * * \\
(-2.75)\end{array}$ \\
\hline Recession $_{\mathrm{t}}$ & $\begin{array}{l}-1.90 \\
(-1.76)\end{array}$ & $\begin{array}{l}-1.87 \\
(-1.76)\end{array}$ & $\begin{array}{l}-1.87 \\
(-1.76)\end{array}$ & $\begin{array}{l}-1.87 \\
(-1.76)\end{array}$ \\
\hline Volatility $_{\mathrm{t}}$ & $\begin{array}{c}-119.95 * * * \\
(-4.63)\end{array}$ & $\begin{array}{c}-118.37 * * * \\
(-4.58)\end{array}$ & $\begin{array}{c}-118.58 * * * \\
(-4.59)\end{array}$ & $\begin{array}{c}-118.45 * * * \\
(-4.59)\end{array}$ \\
\hline $\log \left(\right.$ Market Cap $\left._{t}\right)$ & $\begin{array}{c}-0.06 \\
(-0.52)\end{array}$ & $\begin{array}{c}-0.11 \\
(-0.93)\end{array}$ & $\begin{array}{c}-0.11 \\
(-0.95)\end{array}$ & $\begin{array}{l}-0.11 \\
(-0.93)\end{array}$ \\
\hline Book to Market ${ }_{t}$ & $\begin{array}{l}1.63 * * \\
(2.97)\end{array}$ & $\begin{array}{l}1.56 * * \\
(2.86)\end{array}$ & $\begin{array}{c}1.55 * * \\
(2.83)\end{array}$ & $\begin{array}{c}1.56 * * \\
(2.87)\end{array}$ \\
\hline Beta $_{\mathrm{t}}$ & $\begin{array}{c}0.45 \\
(0.90)\end{array}$ & $\begin{array}{c}0.35 \\
(0.72)\end{array}$ & $\begin{array}{c}0.36 \\
(0.73)\end{array}$ & $\begin{array}{c}0.35 \\
(0.71)\end{array}$ \\
\hline $\begin{array}{l}\Delta \text { Dividend Payout }_{t} \\
\times \log \left({\left.\text { Market } \text { Cap }_{t}\right)}\right)\end{array}$ & & $\begin{array}{c}-2.01 \\
(-0.90)\end{array}$ & & \\
\hline $\begin{array}{l}\Delta \text { Dividend Payout }_{t} \\
\times \log \left({\left.\text { Market } \text { Cap }_{t}\right)} \text { Book to Market }_{t}\right.\end{array}$ & & & $\begin{array}{c}1.32 \\
(0.99)\end{array}$ & \\
\hline
\end{tabular}

All variables are defined in Appendix. 


\begin{tabular}{|c|c|c|c|c|}
\hline $\begin{array}{l}\Delta \text { Dividend Payout }_{\mathrm{t}} \\
\times \log _{\left(\text {Market } \text { Cap }_{\mathrm{t}}\right)} \\
\times \text { Manufacturing }\end{array}$ & & & & $\begin{array}{c}-1.68 \\
(-0.73)\end{array}$ \\
\hline Industry Fixed Effects & Yes & Yes & Yes & Yes \\
\hline Year Fixed effects & Yes & Yes & Yes & Yes \\
\hline Adjusted $\mathrm{R}^{2}$ & 0.02 & 0.01 & 0.01 & 0.01 \\
\hline
\end{tabular}

TABLE 7

\section{DETERMINANTS OF CHANGES IN PE RATIO BY GROUPS}

This table reports the regression coefficients and associated heteroscedasticity consistent $t$-statistics (along with adjusted $\mathrm{R}^{2}$ ) for explaining changes in $\mathrm{P} / \mathrm{E}$ ratio, where changes in dividend payout ratio is the key explanatory variable:

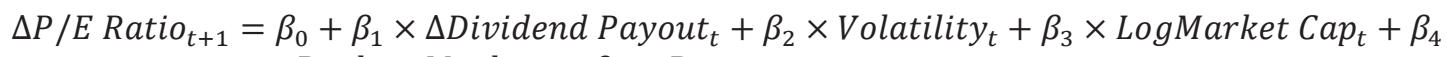

$$
\begin{aligned}
& \times \text { Book to Market }_{t}+\beta_{5} \times \text { Beta }_{t}+\varepsilon
\end{aligned}
$$

The regression is run separately over 90 groups overall, made up of 5 industry groups, 3 groups of market capitalization, 3 groups of book to market values, and whether or not it is a recession period. Only the specifications for which $\Delta$ Dividend Payout is significant at the $5 \%$ and $1 \%$ levels are shown. All variables are defined in Appendix.

\begin{tabular}{|c|c|c|c|c|c|c|c|c|c|}
\hline Industry & $\begin{array}{l}\text { Rank } \\
\text { MVE }\end{array}$ & $\begin{array}{c}\text { Rank } \\
\text { BM }\end{array}$ & REC & $\begin{array}{l}\Delta \text { Dividend } \\
\text { Payout }_{t}\end{array}$ & Volatility $_{t}$ & $\begin{array}{c}\log (\text { Market } \\
\left.\text { Cap }_{t}\right)\end{array}$ & $\begin{array}{l}\text { Book to } \\
\text { Market }_{t}\end{array}$ & Beta $_{t}$ & $\begin{array}{c}\text { Adjusted } \\
\mathrm{R}^{2}\end{array}$ \\
\hline Cnsmr & High & Low & 0 & $\begin{array}{c}-77.8 * * * \\
(-6.2)\end{array}$ & $\begin{array}{l}-152.3 \\
(-1.4)\end{array}$ & $\begin{array}{l}1.5 * \\
(1.8)\end{array}$ & $\begin{array}{c}9.8 \\
(1.5)\end{array}$ & $\begin{array}{l}-4.9 * \\
(-2.1)\end{array}$ & 0.02 \\
\hline Cnsmr & High & Mid & 0 & $\begin{array}{c}-86.6 * * * \\
(-4.3)\end{array}$ & $\begin{array}{l}-62.0 \\
(-0.4)\end{array}$ & $\begin{array}{c}0.1 \\
(0.1)\end{array}$ & $\begin{array}{c}19.0 * \\
(1.7)\end{array}$ & $\begin{array}{c}-1.2 \\
(-0.3)\end{array}$ & 0.02 \\
\hline Cnsmr & Low & Mid & 0 & $\begin{array}{c}-69.5 * * \\
(-3.0)\end{array}$ & $\begin{array}{l}-105.7 \\
(-0.7)\end{array}$ & $\begin{array}{c}-0.3 \\
(-0.1)\end{array}$ & $\begin{array}{c}18.1 * \\
(1.7)\end{array}$ & $\begin{array}{c}1.3 \\
(0.5)\end{array}$ & 0.01 \\
\hline Cnsmr & Mid & Low & 0 & $\begin{array}{c}-100.8 * * * \\
(-4.6)\end{array}$ & $\begin{array}{l}35.9 \\
(0.3)\end{array}$ & $\begin{array}{l}-0.3 \\
(-0.2)\end{array}$ & $\begin{array}{l}11.2 \\
(1.2)\end{array}$ & $\begin{array}{c}0.8 \\
(0.3)\end{array}$ & 0.01 \\
\hline Cnsmr & Mid & Mid & 0 & $\begin{array}{c}-67.7 * * * \\
(-3.6)\end{array}$ & $\begin{array}{l}-172.9 \\
(-1.4)\end{array}$ & $\begin{array}{c}0.5 \\
(0.3)\end{array}$ & $\begin{array}{l}11.1 \\
(1.2)\end{array}$ & $\begin{array}{c}2.8 \\
(1.1)\end{array}$ & 0.01 \\
\hline HiTec & High & Low & 0 & $\begin{array}{c}-67.6 * * \\
(-3.2)\end{array}$ & $\begin{array}{c}-173.8 * \\
(-1.8)\end{array}$ & $\begin{array}{l}-1.3 * \\
(-1.6)\end{array}$ & $\begin{array}{c}23.8 * * \\
(3.0)\end{array}$ & $\begin{array}{c}-1.6 \\
(-0.6)\end{array}$ & 0.01 \\
\hline HiTec & High & Mid & 0 & $\begin{array}{c}-70.2 * * \\
(-2.9)\end{array}$ & $\begin{array}{l}11.5 \\
(0.1)\end{array}$ & $\begin{array}{c}1.6 \\
(1.4)\end{array}$ & $\begin{array}{c}-5.9 \\
(-0.5)\end{array}$ & $\begin{array}{c}1.7 \\
(0.4)\end{array}$ & 0.01 \\
\hline HiTec & Low & Low & 0 & $\begin{array}{c}-126.6 * * \\
(-2.8)\end{array}$ & $\begin{array}{l}-207.4 \\
(-1.2)\end{array}$ & $\begin{array}{c}1.9 \\
(0.5)\end{array}$ & $\begin{array}{l}21.7 \\
(1.2)\end{array}$ & $\begin{array}{l}-4.5 \\
(-1.4)\end{array}$ & 0.01 \\
\hline Hlth & High & Low & 0 & $\begin{array}{c}-126.8 * * * \\
(-5.5)\end{array}$ & $\begin{array}{l}-128.9 \\
(-0.9)\end{array}$ & $\begin{array}{c}0.7 \\
(0.8)\end{array}$ & $\begin{array}{l}13.2 \\
(1.3)\end{array}$ & $\begin{array}{c}0.1 \\
(0.02)\end{array}$ & 0.02 \\
\hline Hlth & Mid & Mid & 1 & $\begin{array}{c}-343.4 * * \\
(-2.9)\end{array}$ & $\begin{array}{l}320.0 \\
(0.5)\end{array}$ & $\begin{array}{l}18.3 \\
(1.5)\end{array}$ & $\begin{array}{l}-12.4 \\
(-0.4)\end{array}$ & $\begin{array}{c}-6.8 \\
(-0.3)\end{array}$ & 0.11 \\
\hline
\end{tabular}




\begin{tabular}{|c|c|c|c|c|c|c|c|c|c|}
\hline Manuf & High & High & 0 & $\begin{array}{c}-81.0 * * * \\
(-5.2)\end{array}$ & $\begin{array}{c}-144.9 \\
(-0.7)\end{array}$ & $\begin{array}{l}-2.3 * \\
(-1.7)\end{array}$ & $\begin{array}{c}4.5 \\
(0.7)\end{array}$ & $\begin{array}{l}6.0 * \\
(1.8)\end{array}$ & 0.03 \\
\hline Manuf & High & Low & 0 & $\begin{array}{c}-66.3 * * * \\
(-5.2)\end{array}$ & $\begin{array}{c}-353.5 * * * \\
(-3.9)\end{array}$ & $\begin{array}{c}0.3 \\
(0.4)\end{array}$ & $\begin{array}{c}16.5 * \\
(2.2)\end{array}$ & $\begin{array}{l}3.8 * \\
(2.1)\end{array}$ & 0.02 \\
\hline Manuf & High & Mid & 0 & $\begin{array}{c}-65.5 * * * \\
(-4.2)\end{array}$ & $\begin{array}{c}-230.9 * \\
(-1.6)\end{array}$ & $\begin{array}{l}-0.5 \\
(-0.6)\end{array}$ & $\begin{array}{c}18.1 * \\
(2.0)\end{array}$ & $\begin{array}{c}1.7 \\
(0.8)\end{array}$ & 0.02 \\
\hline Manuf & Low & Mid & 0 & $\begin{array}{c}-93.6 * * * \\
(-4.6)\end{array}$ & $\begin{array}{l}-56.4 \\
(-0.4)\end{array}$ & $\begin{array}{l}-2.3 \\
(-1.2)\end{array}$ & $\begin{array}{l}11.0 \\
(1.1)\end{array}$ & $\begin{array}{l}0.712 \\
(0.3)\end{array}$ & 0.02 \\
\hline Manuf & Mid & High & 0 & $\begin{array}{c}-114.3 * * * \\
(-5.7)\end{array}$ & $\begin{array}{l}125.9 \\
(0.7)\end{array}$ & $\begin{array}{l}-2.9 \\
(-1.2)\end{array}$ & $\begin{array}{c}1.3 \\
(0.2)\end{array}$ & $\begin{array}{l}1.2 \\
(0.4)\end{array}$ & 0.03 \\
\hline Manuf & Mid & Low & 0 & $\begin{array}{c}-50.5 * * \\
(-2.6)\end{array}$ & $\begin{array}{c}-355.0 * * \\
(-2.7)\end{array}$ & $\begin{array}{l}-1.4 \\
(-0.8)\end{array}$ & $\begin{array}{c}7.9 \\
(0.9)\end{array}$ & $\begin{array}{l}0.71 \\
(0.3)\end{array}$ & 0.01 \\
\hline Manuf & Mid & Mid & 0 & $\begin{array}{c}-72.0 * * * \\
(-5.1)\end{array}$ & $\begin{array}{l}-25.3 \\
(-0.2)\end{array}$ & $\begin{array}{l}-0.02 \\
(-0.0)\end{array}$ & $\begin{array}{c}21.5 * * \\
(3.1)\end{array}$ & $\begin{array}{c}2.0 \\
(1.2)\end{array}$ & 0.02 \\
\hline Other & High & High & 0 & $\begin{array}{c}-60.7 * * * \\
(-5.9)\end{array}$ & $\begin{array}{l}-55.0 \\
(-0.6)\end{array}$ & $\begin{array}{l}-0.5 \\
(-0.8)\end{array}$ & $\begin{array}{l}-2.0 \\
(-0.8)\end{array}$ & $\begin{array}{l}3.0 \\
(1.6)\end{array}$ & 0.02 \\
\hline Other & High & Low & 0 & $\begin{array}{c}-83.4 * * * \\
(-5.2)\end{array}$ & $\begin{array}{c}-156.9 \\
(-1.5)\end{array}$ & $\begin{array}{c}-0.8 \\
(-1.1)\end{array}$ & $\begin{array}{c}6.7 \\
(1.0)\end{array}$ & $\begin{array}{c}-0.1 \\
(-0.0)\end{array}$ & 0.02 \\
\hline Other & High & Mid & 0 & $\begin{array}{c}-71.6 * * * \\
(-6.0)\end{array}$ & $\begin{array}{l}-98.1 \\
(-1.3)\end{array}$ & $\begin{array}{l}-0.6 \\
(-1.1)\end{array}$ & $\begin{array}{c}15.5 * * \\
(3.1)\end{array}$ & $\begin{array}{c}0.6 \\
(0.4)\end{array}$ & 0.02 \\
\hline Other & Low & High & 0 & $\begin{array}{c}-70.9 * * * \\
(-5.1)\end{array}$ & $\begin{array}{l}-28.2 \\
(-0.3)\end{array}$ & $\begin{array}{l}1.1 \\
(0.7)\end{array}$ & $\begin{array}{l}-5.0 \\
(-1.5)\end{array}$ & $\begin{array}{c}0.2 \\
(0.1)\end{array}$ & 0.01 \\
\hline Other & Mid & High & 0 & $\begin{array}{c}-53.9 * * * \\
(-4.6)\end{array}$ & $\begin{array}{l}-78.8 \\
(-0.9)\end{array}$ & $\begin{array}{c}0.4 \\
(0.4)\end{array}$ & $\begin{array}{l}5.2 * \\
(1.8)\end{array}$ & $\begin{array}{l}-3.0 * \\
(-1.8)\end{array}$ & 0.01 \\
\hline Other & Mid & Low & 0 & $\begin{array}{c}-81.7 * * * \\
(-3.6)\end{array}$ & $\begin{array}{c}-140.9 \\
(-1.2)\end{array}$ & $\begin{array}{l}-1.8 \\
(-0.9)\end{array}$ & $\begin{array}{l}-1.3 \\
(-0.1)\end{array}$ & $\begin{array}{c}-0.5 \\
(-0.2)\end{array}$ & 0.01 \\
\hline Other & Mid & Mid & 0 & $\begin{array}{c}-50.6 * * * \\
(-3.5)\end{array}$ & $\begin{array}{c}-174.7 * \\
(-2.3)\end{array}$ & $\begin{array}{l}-1.2 \\
(-1.1)\end{array}$ & $\begin{array}{c}12.8 * \\
(2.5)\end{array}$ & $\begin{array}{l}1.0 \\
(0.7)\end{array}$ & 0.01 \\
\hline
\end{tabular}

$*, * *$, and $* * *$ denote significant at the $10 \%, 5 \%$ and $1 \%$ level respectively. 


\section{TABLE 8 \\ DETERMINANTS OF RISK}

This table reports the regression coefficients and the associated heteroscedasticity consistent tstatistics (along with adjusted $\mathrm{R}^{2}$ ) for explaining change in stock return volatility (standard deviation) in the next period, where change in dividend payout ratio of the current period is the main explanatory variable.

$$
\begin{aligned}
& \Delta \text { Volatility }_{t+1}= \beta_{0}+\beta_{1} \times \Delta \text { Dividend Payout }_{t}+\beta_{2} \times \text { LogMarket Cap }_{t}+\beta_{3} \times{\text { Book to } \text { Market }_{t}+}+ \\
& \beta_{4} \times \text { Beta }_{t}+\varepsilon
\end{aligned}
$$

\begin{tabular}{|c|c|}
\hline & $\Delta$ Volatility $_{t+1}$ \\
\hline$\Delta$ Dividend Payout $t_{t}$ & $\begin{array}{c}0.001 * * * \\
(4.0)\end{array}$ \\
\hline $\log \left(\right.$ Market Cap $\left._{t}\right)$ & $\begin{array}{c}0.001 * * * \\
(4.6)\end{array}$ \\
\hline Book to Market $_{t}$ & $\begin{array}{c}-0.001 \\
(-1.4)\end{array}$ \\
\hline Beta $_{\mathrm{t}}$ & $\begin{array}{c}-0.001 * * * \\
(-22.2)\end{array}$ \\
\hline Adjusted $\mathrm{R}^{2}$ & 0.08 \\
\hline
\end{tabular}

All variables are defined in Appendix.

\section{CONCLUSION}

Using a large database of all S\&P 1500 index firms spanning the 88-quarter period from 1995 through 2016, we document that in terms of dividend payout, in average, manufacturing firms payout more, large firms payout more, and firms with lower future growth potential payout more. In terms of PE ratios, in univariate tests, we find that, on average, high tech and healthcare firms have higher PE ratios, and manufacturing firms the least, perhaps reflecting future growth potential. The higher the market capitalization, the higher the PE ratio; and the lower the book-to-market ratio the higher the PE ratio. So, payout and $\mathrm{PE}$ ratios move in opposite directions when we compare by industry, in the same direction when we examine by market cap, and in the opposite direction when we examine by book-to-market ratio, consistent with a perceived growth potential story. In multivariate regressions, we find that current period dividend payout is positively and significantly related to next period PE (at $1 \%$ significance level) after controlling of the year and industry effects. After controlling for other factors, future PE ratio is significantly and negatively associated with market capitalization and higher book to market ratio. Results are consistently significant when we use current earnings or trailing earnings.

After we run regressions according to groups divided by industries, market cap, book-to market ratio and whether or not it is a recession period, we find that, for consumer and high technology industries, the correlation between current period payout and next period PE tends to be significantly negative, while it is significantly positive for manufacturing and other industries. In general, for high market cap firms, the relation between payout and future $\mathrm{PE}$ is positive.

When we examine changes, future PE change is significantly and negatively associated with current period change in payout. We also find that current period payout change is associated with future increased risk. This implies that future PE decreases consistent with increased risk likely due to perceived lack of growth options. In summary, any model of the determinants of PE is complicated, and should take into account industry, size, risk and market perception of future growth for a firm. 


\section{REFERENCES}

Ackert, L. F., \& Hunter, W. C. (2001). An Empirical Examination of the Price-Dividend Relation with Dividend Management. Journal of Financial Services Research, 115-129.

Ang, A., \& Zhang, X. (2011). Price-Earnings Ratios: Growth and Discount Rates. New York: Columbia Business School.

Arnott, R. D., \& Asness, C. S. (2003). Surprise! Higher Dividends = Higher Earnings Growth. Financial Analysts Journal, 70-87.

Eaton, R. V. (1999). Stock Price Adjustment to the Information in Dividend Changes. Review of Quantitative Finance and Accounting, 113-133.

Ferson, W., (2008), Stock Return Predictability, The New Palgrave Dictionary of Economics, 2nd edition, 2008. edited by Steven N. Durlauf and Lawrence E. Blume.

Grullon, G., Michaely, R., \& Swaminathan, B. (2002). Are Dividend Changes a Sign of Firm Maturity? Journal of Business, 3, 387-424.

Henne, A., Ostrowski, S., \& Reichling, P. (2009). Dividend yield and stability versus performance on the German stock market: a descriptive study. Springer, 225-248.

Hough, J. (2011, October 9). Peeling Back the Market's P/E. Wall Street Journal: Retrieve from https://www.wsj.com/articles/SB10001424053111904491704576573241520154756

Penman, S. H. (1996). The Articulation of Price-Earnings Ratios and Market-to-Book Ratios and the Evaluation of Growth. Journal of Accounting Research, 34(2), 235-259.

Riahi-Belkaoui, A., \& Picur, R. D. (2001). Investment Opportunity Set Dependence of Dividend Yield and Price Earnings Ratio. Managerial Finance, 27(3), 65-71.

Shiller, R. J. (1980). Do Stock Prices Move Too Much to be Justified by Subsequent Changes in Dividends? . National Bureau of Economic Reserach, 1-40.

Singh, S., Jain, P., \& Yadav, S. S. (2016). Analysis of Price Multiples. In S. Singh, P. Jain, \& S. S. Yadav, India Studies in Business and Economics, 127-143, New Delhi, India: Springer Nature.

Yan, R., \& Xie, C. (2012). Industry Stock Price Effect and Its Influencing Factors of Cash Dividend Distribution: Based on Chinese Real Estat Listed Companies. In J. Kacprzyk, Advances in Intelligent and Soft Computing, 463-469, Heidelberg: Springer. 


\section{APPENDIX}

\section{VARIABLES DESCRIPTIONS}

\begin{tabular}{|c|c|c|}
\hline Variable & Description & Formula/ Source \\
\hline IBADJQ & $\begin{array}{c}\text { Income Before Extraordinary Items - } \\
\text { Adjusted for Common Stock } \\
\text { Equivalents }\end{array}$ & Compustat \\
\hline NIQ & Net Income (Loss) & Compustat \\
\hline ATQ & Assets - Total & Compustat \\
\hline CEQQ & Common/Ordinary Equity - Total & Compustat \\
\hline LTQ & Liabilities - Total & Compustat \\
\hline PSTKQ & $\begin{array}{l}\text { Preferred/Preference Stock (Capital) } \\
\text { - Total }\end{array}$ & Compustat \\
\hline SEQQ & Stockholders Equity Quarterly & Compustat \\
\hline TXDITCQ & $\begin{array}{c}\text { Deferred Taxes and Investment Tax } \\
\text { Credit }\end{array}$ & Compustat \\
\hline DVY & Cash Dividends & Compustat \\
\hline DVPSPQ & Dividends per Share- Quarter & Compustat \\
\hline PRCCQ & Price Close - Quarter & Compustat \\
\hline CSHOQ & Common Shares Outstanding & Compustat \\
\hline BEQ & Book Value of Equity & $\begin{array}{l}=\text { SEQQ + TXDITCQ - PSTKQ, } \\
\text { If SEQQ missing, SEQQ = CEQQ + PSTKQ, } \\
\text { If also missing, SEQQ = ATQ - LTQ }\end{array}$ \\
\hline DIV & Cash Dividend & $=\max (\operatorname{Delta}(\mathrm{DVY}), \mathrm{DVPSPQ} * \mathrm{CSHOQ})$ \\
\hline Volatility & Volatility & $\begin{array}{l}\text { Previous one year of the DATADATE of stock return } \\
\text { standard deviation }\end{array}$ \\
\hline Beta & Daily past Beta & $\begin{array}{l}\text { Previous one year daily beta by running: } \mathrm{RET}-\mathrm{RF}= \\
\text { alpha }+ \text { beta } * \text { MKTRF }\end{array}$ \\
\hline EPS0 & Earnings per Share & $=$ NIQ / CSHOQ \\
\hline EPSt & Trailing EPS & $=\operatorname{avg}(\mathrm{NIQ}) / \mathrm{CSHOQ}$ for past four quarters \\
\hline PE0 & Price/Earnings Ratio & $=$ PRCCQ $/$ EPS0 \\
\hline PEt & Price/Trailing Earnings Ratio & $=$ PRCCQ / EPSt \\
\hline Dividend Payout & Dividend Payout Ratio & $=\mathrm{DIV} / \mathrm{IBADJQ}$ if IBADJQ $>0$ \\
\hline PE01 & $\mathrm{P} / \mathrm{E}$ Ratio for $\mathrm{t}+1$ & $=$ lead(PE0) \\
\hline PE02 & $\mathrm{P} / \mathrm{E}$ Ratio for $\mathrm{t}+2$ & $=$ lead $(\mathrm{PE} 01)$ \\
\hline PEt1 & Trailing P/E Ratio for $\mathrm{t}+1$ & $=$ lead $(\mathrm{PEt} 0)$ \\
\hline PEt2 & Trailing P/E Ratio for $\mathrm{t}+2$ & $=$ lead(PEt1) \\
\hline $\begin{array}{l}\text { DPR_1 } \\
\text { Industry }\end{array}$ & $\begin{array}{l}\text { Dividend Payout for t-1 } \\
\text { Fama French } 5 \text { Industry } \\
\text { classification }\end{array}$ & $\begin{array}{l}=\operatorname{lag}(\mathrm{DPR}) \\
\text { Cnsmr, HiTec, Hlth, Manuf, Other }\end{array}$ \\
\hline MVE & $\begin{array}{l}\text { Market Value of Equity or Market } \\
\text { cap }\end{array}$ & $=\mathrm{PRCCQ} * \mathrm{CSHOQ}$ \\
\hline BMR & Book to Market Ratio & $=\mathrm{BEQ} / \mathrm{MVE}$ \\
\hline Rank MVE & Ranking by MVE & $\begin{array}{l}\text { Divide each quarter's MVE into } 3 \text { equal groups: } \\
\text { "High", "Mid", "Low" }\end{array}$ \\
\hline Rank BMR & Ranking by BMR & $\begin{array}{l}\text { Divide each quarter's BMR into } 3 \text { equal groups: } \\
\text { "High", "Mid", "Low" }\end{array}$ \\
\hline
\end{tabular}

